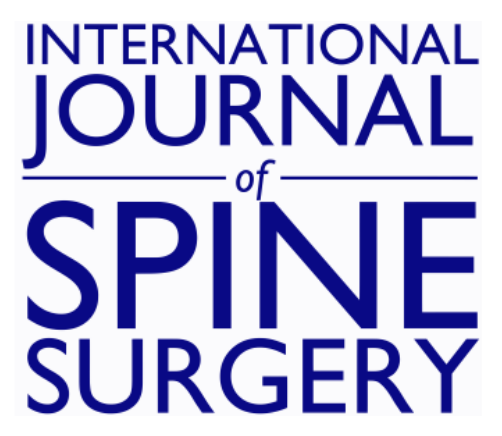

\title{
Idiopathic spinal cord herniation with duplicated dura mater and dorsal subarachnoid septum. Report of a case and review of the literature
}

Norio Yamamoto, Shinsuke Katoh, Kousaku Higashino and Koichi Sairyo

Int J Spine Surg 2014, 8 ()

doi: https://doi.org/10.14444/1029

http://ijssurgery.com/content/8/29

This information is current as of April 26, 2023.

Email Alerts Receive free email-alerts when new articles cite this article. Sign up at:

http://ijssurgery.com/alerts

The International Journal of Spine Surgery

2397 Waterbury Circle, Suite 1,

Aurora, IL 60504, Phone: +1-630-375-1432 


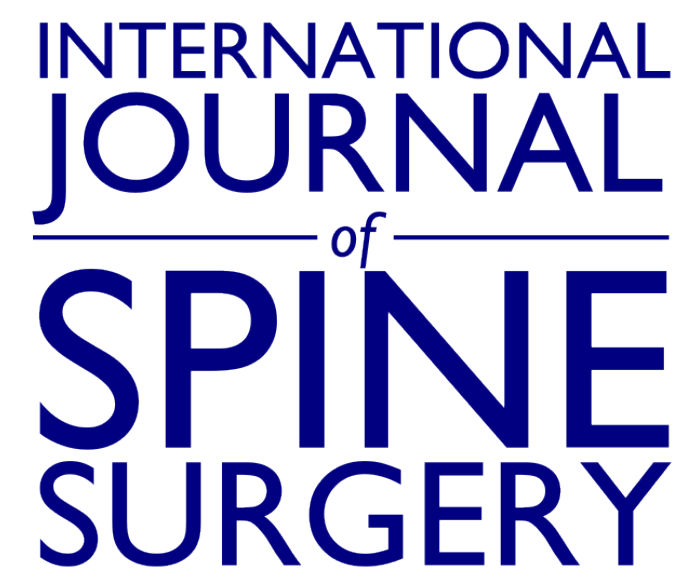

This article generously published free of charge by the International Society for the Advancement of Spine Surgery.

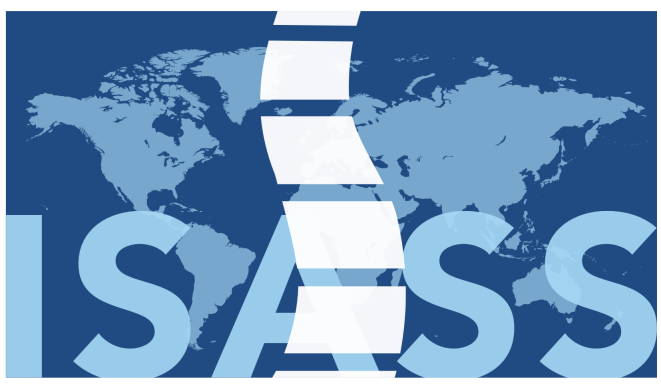

INTERNATIONAL SOCIETY for the ADVANCEMENT of SPINE SURGERY 


\section{Idiopathic spinal cord herniation with duplicated dura mater and dorsal subarachnoid septum. Report of a case and review of the literature.}

Norio Yamamoto, MD, ${ }^{1}$ Shinsuke Katoh, MD, PhD, ${ }^{2}$ Kousaku Higashino MD,PhD, ${ }^{1}$ Koichi Sairyo, $M D, P h D^{l}$

${ }^{1}$ Department of Orthopedics, Institute of Health Biosciences, The University of Tokushima Graduate School, Tokushima, Japan ${ }^{2}$ Department of Rehabilitation, Tokushima University

Hospital, Tokushima, Japan

\section{Abstract}

\section{Background}

Idiopathic spinal cord herniation (ISCH) is a rare condition and its pathogenesis remains unclear. The purpose of this case report is to present an ISCH case with dorsal subarachnoid septum suggesting the pathogenesis of ISCH being adhesions from preexisting inflammation.

\section{Methods}

Single case report.

\section{Results}

A 60-year-old woman presented with Brown-Séquard syndrome below the level of T6. Magnetic resonance imaging revealed the thoracic spinal cord was displaced ventrally, and the dorsal subarachnoid space was enlarged and had a septum between the spinal cord and dura mater. Intraoperatively, the dorsal dura mater was seen to be adherent and the subarachnoid septum was identified after durotomy. The inner layer defect of the duplicated dura mater was found in the ventral dura mater, through which the spinal cord had herniated. After releasing the septum, the adhesions around the dura mater, and the hiatus, the spinal cord was reduced.

\section{Conclusions}

The present case indicates that adhesions around the dura mater can be the pathogenesis of ISCH. 


\section{Introduction}

Wortzman et $\mathrm{al}^{1}$ reported the first case of idiopathic spinal cord herniation (ISCH) in 1974. In the years since magnetic resonance imaging (MRI) came into widespread use, the concept of ISCH has been gradually appreciated, and the number of reported cases has increased since 1990. This herniation exclusively occurs in the thoracic spine, typically $\mathrm{T} 4-\mathrm{T} 7,{ }^{2}$ causing progressive myelopathy. Diagnosis is based on ventral displacement of the spinal cord observed on MRI and CT myelography.

Aizawa et $\mathrm{al}^{3}$ classified this condition into three types depending on the abnormality of the ventral portion of the dura mater, but the pathogenesis of ISCH has yet to be established. Both congenital and acquired mechanisms have been postulated for development of the lesion. ${ }^{2}$ An adhesions secondary to inflammation of the dura mater is one candidate. Here we report a case of ISCH with a dorsal subarachnoid septum suggesting preexisting inflammation as the pathogenesis.

\section{Case report}

\section{History and examination}

A 60-year-old active woman presented with a loss of temperature and pinprick sensation in her left leg, a loss of tactile sensation and muscle weakness of the right lower leg at the age of 45. She had no history of spinal surgery, spinal puncture, or trauma. Her symptoms slowly progressed, and on presentation the gait was spastic, especially on the right side. Neurological examination revealed Brown-Séquard syndrome below the level of T6. Position and vibration sensations were preserved bilaterally. Hyperreflexia and ankle clonus were observed of the right leg. Muscle strength was reduced to 4/5 (moves joint against gravity and resistance but no normal strength) on the right and normal on the left. Vesicorectal functions were preserved. Peripheral blood tests, biochemical tests, chest Xray and urinalysis revealed no abnormalities.

\section{Radiological findings}

MRI showed the thoracic spinal cord displaced ventrally to the right, an enlarged dorsal subarachnoid space, and a midline septum between the dura mater and spinal cord at T5-T6 disc level (Figure 1). The midline septum separated dorsal subarachnoid space at the level, but not cranial nor caudal levels. Spinal cord was flattened, and high T2 signal intensity was not detected. Ventral spinal cord deviation was limited at T5-T6 disc level. Scalloping of the vertebral body ${ }^{4}$ and erosion of the dura mater by a herniated and calcified disc ${ }^{5}$ were not seen. CT myelography showed the compressed spinal cord ventrally as well as no pooling and extravasation of contrast medium ventral to the spinal cord. As for dorsal side of spinal cord, it also showed an uninterrupted flow of contrast material and no filling defect (Figure 2). A nuclear trail sign, a feature suggestive of a path of herniation of the nucleus pulposus, was not present. The septum seen on MRI could not be distinguished with contrast medium on CT myelography. 


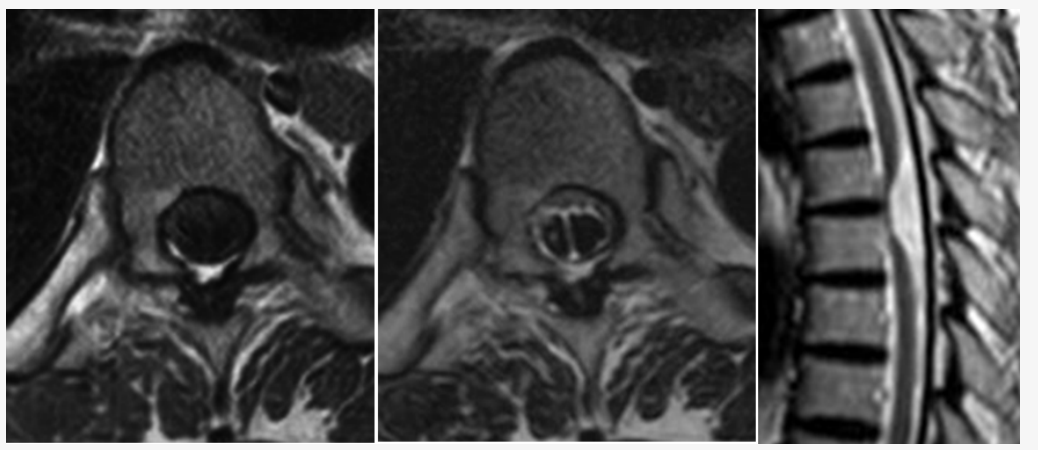

Fig. 1. Axial T1, T2-weighted MRI (left, center) shows significant right ventral shift of the spinal cord and a dorsal midline subarachnoid septum at T5-T6 disc level. The midline septum is observed only at this level. Sagittal T2-weighted MRI (right) shows ventral

displacement of the spinal cord at T5-T6 disc level. Focal ventral kinking and adhesion of the spinal cord is apparent with an enlarged dorsal subarachnoid space.

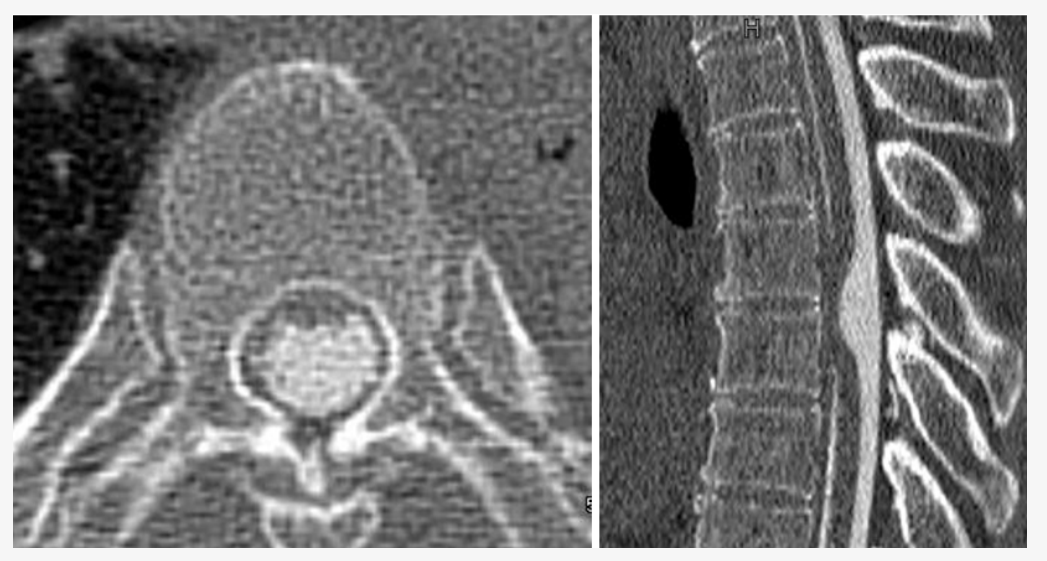

Fig. 2. Axial CT myelography at the same level as the MRI axial image (Figure 1) shows no pooling of contrast medium ventrally to the spinal cord, an uninterrupted flow of contrast material and no filling defect.

\section{Operative findings}

Laminectomy and right-sided partial pediculotomy of the T5 and T6 vertebrae was performed, and adhesion of the dura mater was found. Ultrasonography revealed the septum in the dorsal subarachnoid space, and a ventral spinal cord herniation and the dorsal subarachnoid septum were identified after durotomy. The septum was a muddy, thin, shiny whitish membrane containing small vessels, and was $15 \mathrm{~mm}$ in cephalocaudal length. It connected the dorsal dura mater to the dorsal surface of the spinal cord (Figure 3 ), it is not a normal arachnoid trabeculation which is usually seen in the thoracic spine. Cerebrospinal fluid (CSF) flowed freely around the septum. 

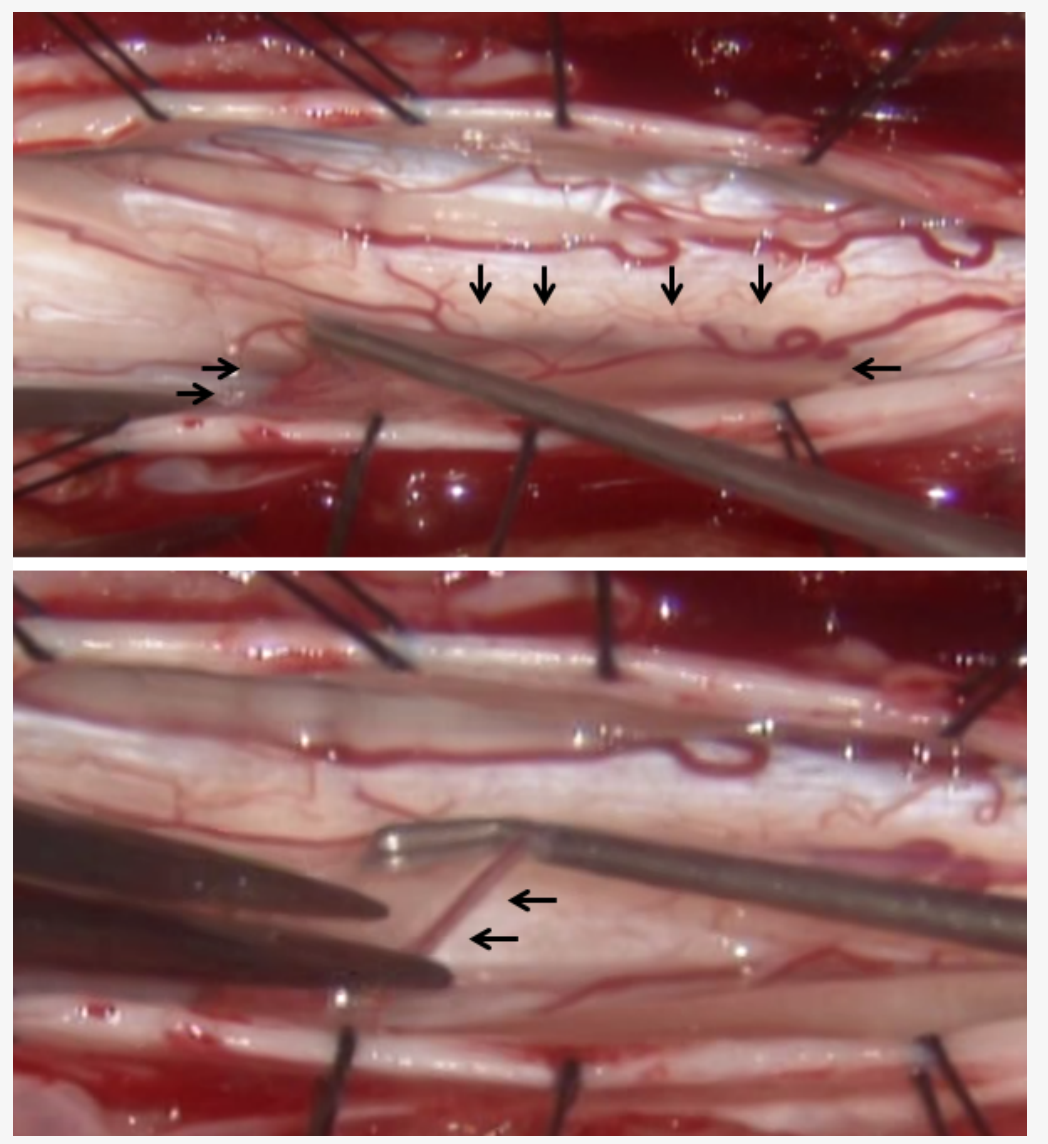

Fig. 3. Intraoperative photographs. The upper photograph shows the dorsal subarachnoid septum (arrows). Right side is cranial. The lower photograph indicates the vessels in the septum (arrows).

After removal of the septum, the spinal cord was not reduced into the normal position. The dentate ligament was divided, the defect of the inner layer of the ventral dura mater was found, through which the spinal cord had herniated. Incarceration of the spinal cord in the hernia orifice was not observed. The herniated hiatus was enlarged by a $15 \mathrm{~mm}$ cephalocaudal incision and the spinal cord was carefully untethered without significant traction. Through the defect, another glossy white layer of dura mater, which was presumed to be the outer layer of the duplicated dura mater, was observed. With ultrasonography, we confirmed that CSF flowed freely in the ventral arachnoid space and the spinal cord had reduced.

\section{Postoperative course}

By 2 postoperative weeks, the patient had recovered muscle power and some sensation, with a slight lessening of spasticity. A half year later, the spinal cord remained reduced on postoperative MRI (Figure 4). Atrophy and high signal changes within the spinal cord were not observed. At 2 years follow up, the patient had no recurrence of symptoms, no instability and no back pain. 


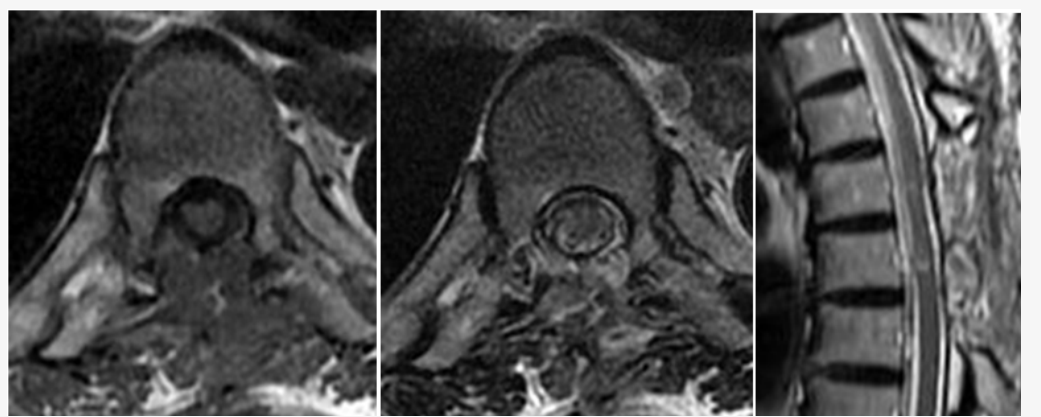

Fig. 4. Axial T1, T2-weighted MRI (left, center), sagittal T2-weighted MRI (right). The focal mildly high T2 intensity in the cord (arrows) was believed to be due to regional edema, gliosis or postoperative effects.

\section{Discussion}

ISCH typically occurs in middle-aged adults with a female preponderance. A slowly progressive Brown-Séquard syndrome at upper thoracic spine is the most common clinical presentation. All considered, it seems more likely that ISCH is a pathological condition acquired in adulthood. An inflammation and degenerative process with slow erosion of the dura mater would seem consistent with the age of presentation and the location of lesion.

The proposed pathogenesis of ISCH is initiated by minor or unrecognized trauma and inflammation including mechanical stress, which means CSF pulsation, physiological anterior movement of spinal cord secondary to cardiac pulsations and respiratory movements, and the biomechanical impact of flexion and extension movements at thoracic spine, although active inflammation may not be present in the inner layer of dura mater. ${ }^{6}$ The thoracic spine, where spinal cord is anteriorly located and the change in spinal fluid flow occurs, ${ }^{7}$ has limited mobility, physiologic kyphosis at this level, which may lead to ventral adhesion of the pia mater once inflammation develops around the dura mater. Dorsally thickened arachnoid mater has also been reported by many authors and this adhesion seems to be secondary to inflammation of the dura mater. ${ }^{8}$ Prada et al. reported that histology of thick, fibrotic, inflammatory tissue surrounding the herniated cord demonstrated a chronic dural inflammatory process. ${ }^{9}$ Therefore, it may be that the inflammatory reactions causing adhesion of the arachnoid mater occur in the early pathogenesis of ISCH and then ventral adhesion of the spinal cord occurs. ${ }^{10}$ Together with cardiorespiratory-associated CSF pulsations, this event may cause continuous spinal cord friction and erosion of the dura mater, which could lead to the formation of the dural defect with progressive herniation of the spinal cord. ${ }^{11}$ Unfortunately for this case, we could not perform histologic examination of the septum and the inner layer of duplicated dura mater because it was too thin to capture without destructing the original form. Several authors reported that histologic examinations demonstrated that the inner layer of duplicated dura mater consisted of fibrous connective tissue without inflammatory cellular infiltration. ${ }^{3}$ 
One important lesion in the differential diagnosis of this condition is compressive dorsal arachnoid cyst ${ }^{12}$ which is the most common erroneous radiological diagnosis in patients with ISCH. Our case showed no filling defect by CT myelography, which was confirmed during surgery.

In our case, we speculate that circumferential adhesion had occurred early in disease pathogenesis, the dorsal adhesion was released by pulsations of CSF, the spinal cord was tethered ventrally, a part of the adhesion remained which became the dorsal subarachnoid septum, and the ventral adhesion caused ISCH. The definitive diagnoses can be made only with intraoperative findings. ${ }^{11}$ In case of a suspected dorsal subarachnoid septum, the surgeon should proceed to thoroughly inspect the ventral portion of spinal cord and dura mater.

\section{Conclusion}

Idiopathic spinal cord herniation is rare, but is an increasingly recognized cause of progressive and potentially curable thoracic myelopathy. The present case indicates that adhesions around the dura mater can be the pathogenesis of ISCH.

\section{References}

1. Wortzman G, Tasker RR, Rewcastle NB, Richardson JC, Pearson FG. Spontaneous incarcerated herniation of the spinal cord into a vertebral body: a unique cause of paraplegia. Case report. J Neurosurg. 1974;41:631-5.

2. Tekkok IH. Spontaneous spinal cord herniation: case report and review of the literature. Neurosurgery 2000;46:485-91

3. Aizawa T, Sato T, Tanaka Y, Kotajima S, Sekiya M, Kokubun S. Idiopathic herniation of the thoracic spinal cord: report of three cases. Spine 2001;26:E488-91.

4. Barbagallo GM, Marshman LA, Hardwidge C, Gullan RW. Thoracic idiopathic spinal cord herniation at the vertebral body level: a subgroup with a poor prognosis? Case reports and review of the literature. J Neurosurg. 2002;97:369-74.

5. Brus-Ramer M, Dillon WP. Idiopathic thoracic spinal cord herniation: retrospective analysis supporting a mechanism of diskogenic dural injury and subsequent tamponade. AJNR Am J Neuroradiol. 2012; 33:52-6.

6. Borges LF, Zervas NT, Lehrich JR. Idiopathic spinal cord herniation: a treatable cause of the Brown-Sequard syndrome--case report. Neurosurgery 1995;36:1028-32

7. Abe M, Komori H, Yamaura I, Kayano T. Spinal cord herniation into an extensive extradural meningeal cyst: postoperative analysis of intracystic flow by phasecontrast cine MRI. J Orthop Sci 1999;4:450-6.

8. Sugimoto T, Kasai Y, Takegami K, Morimoto R, Maeda M, Uchida A. A case of idiopathic spinal cord herniation with duplicated dura mater. J Spinal Disord Tech 2005;18:106-11.

9. Prada F, Saladino A, Giombini S, Erbetta A, Saini M, Dimeco F, et al. Spinal cord herniation: Management and outcome in a series of 12 consecutives patients and review of the literature. Acta Neurochir (Wien) 2012;154:723-30

10. Najjar MW, Baeesa SS, Lingawi SS. Idiopathic spinal cord herniation: a new theory of pathogenesis. Surg Neurol 2004;62:161-70 
11. Kumar R, Taha J, Greiner AL. Herniation of the spinal cord. Case report. J Neurosurg 1995;82:131-6.

12. Isu T, Iizuka T, Iwasaki Y, Nagashima M, Akino M, Abe H. Spinal cord herniation associated with an intradural spinal arachnoid cyst diagnosed by magnetic resonance imaging. Neurosurgery 1991;29:137-9.

\section{Corresponding Author}

S. Katoh, MD, PhD, Department of Rehabilitation, Tokushima University Hospital, 3-18-15 Kuramoto, Tokushima 770-8503, Japan. skatoh@clin.med.tokushima-u.ac.jp

\section{Disclosures}

The authors declare no financial disclosures.

Copyright (C) 2014 ISASS - International Society for the Advancement of Spine Surgery. To see more or order reprints or permissions, see http://ijssurgery.com. 